\title{
Band-structure topologies of graphene: spin-orbit coupling effects from first principles
}

\author{
M. Gmitra ${ }^{1}$, S. Konschuh ${ }^{1}$, C. Ertler ${ }^{1}$, C. Ambrosch-Draxl ${ }^{2}$ and J. Fabian ${ }^{1}$ \\ ${ }^{1}$ Institute for Theoretical Physics, University of Regensburg, 93040 Regensburg, Germany \\ ${ }^{2}$ Chair or Atomistic Modeling and Design of Materials, \\ University of Leoben, Franz-Josef-Strasse 18, A-8700 Leoben, Austria
}

\begin{abstract}
The electronic band structure of graphene in the presence of spin-orbit coupling and transverse electric field is investigated from first principles using the linearized augmented plane-wave method. The spin-orbit coupling opens a gap at the $K\left(K^{\prime}\right)$-point of the magnitude of $24 \mu \mathrm{eV}(0.28 \mathrm{~K})$. This intrinsic splitting comes $96 \%$ from the usually neglected $d$ and higher orbitals. The electric field induces an additional (extrinsic) Bychkov-Rashba-type splitting of $10 \mu \mathrm{eV}(0.11 \mathrm{~K}) \mathrm{per} \mathrm{V} / \mathrm{nm}$, coming from the $\sigma-\pi$ mixing. A 'mini-ripple' configuration with every other atom is shifted out of the sheet by less than $1 \%$ differs little from the intrinsic case.
\end{abstract}

The fascination with graphene [1], the one-atom-thick allotrope of carbon, comes from its two-dimensional structure as well as from its unique electronic properties $[2,3,44,5,6]$. The latter originate from the specific electronic band structure at the Fermi level: electrons move with a constant velocity, apparently without mass and a spectral gap. Analogy with massless Dirac fermions is often drawn, presenting graphene as a solid-state toy for relativistic quantum mechanics. Ironically, this nice analogy is broken by the relativistic effects themselves. In particular, the interaction of the orbital and spin degrees of freedom, spin-orbit coupling, gives the electrons in graphene a finite mass and induces a gap in the spectrum. How large is the gap and which orbital states contribute to it? This question is crucial for knowing graphene's band-structure topology, understanding its spin transport and spin relaxation properties [7, 8], or for assessing prospects of graphene for spin-based quantum computing [9]. By performing comprehensive first-principles calculations we predict the spectral gap and establish the relevant electronic spectrum of graphene in the presence of external transverse electric field. We find that realistic electric fields can tune among different band structure topologies with important ramifications for the physics of graphene.

Carbon atoms in graphene are arranged in a honeycomb lattice which comprises two triangular Bravais lattices; the unit cell has two atoms. The corresponding reciprocal lattice is again honeycomb, with two nonequivalent vertices $K$ and $K^{\prime}$ which are the Fermi momenta of a neutral graphene. The states relevant for transport are concentrated in two touching cones with the tips at $K\left(K^{\prime}\right)$ - the Dirac points - as illustrated in Fig. 1. The corresponding Bloch states are formed mainly by the carbon valence $p_{z}$ orbitals (the $\mathrm{z}$-axis is perpendicular to the graphene plane) forming the two $\pi$ bands (cones). The other three occupied valence states of carbon form the deep-lying $\sigma$ bands by $s p^{2}$ hybridization; these are responsible for the robustness of graphene's structure. The states in the lower cones are hole or valence like, the upper cone states are electron or conduction like, borrowing from semiconductor terminology. These essentials of the electronic band structure of graphene were worked out many decades ago [10, 11, 12, 13, 14, 15].

The above picture breaks down when spin-orbit coupling is included. The most important modification to the band structure is the opening of a gap at $K\left(K^{\prime}\right)$, as predicted by Slonczewski and Weiss [14, 16]. More severe changes occur when graphene is subject to transverse electric field that can come from the substrate or electric gates. Several questions arise: (i) What is the gap caused by the spin-orbit coupling itself? The magnitude of this intrinsic gap gives the temperature scale for observing the spin-orbit effects. While the spin-orbit coupling splitting of the $p$-orbitals in carbon atoms is of order $10 \mathrm{meV}$, the relevant states in graphene comprise $p_{z}$ orbitals which have no net orbital momentum along $z$ so the effect is expected to be rather weak. (ii) For realistic electric fields, what are the corresponding extrinsic effects on the band structure? Are the effects comparable to the intrinsic ones? The answer helps to decide on the spin transport mode as well as on spin relaxation and spin coherence mechanisms, or to see if the band structure can be tailored. (iii) Which orbitals are involved in the intrinsic and extrinsic effects? Finally, (iv) do transverse deformations of the graphene plane induce significant spin-orbit effects?

To investigate the electronic band structure of graphene in the presence of spin-orbit coupling and to answer questions (i) to (iv) we employed the full potential linearized augmented plane waves (LAPW) method based on density functional theory [17]. For exchangecorrelation effects we used the generalized gradient approximation (GGA) [18]. In our three-dimensional calculation the graphene sheets of lattice constant $a=$ $1.42 \sqrt{3} \AA$ are separated by the distance $20 \AA$, large enough for the inter-sheet tunneling to be negligible. Integration in the reciprocal space was performed by the modified Blöchl tetrahedron scheme, taking the mesh of $33 \times 33 k$-points in the irreducible Brillouin zone wedge. As the plane-wave cut-off we took $9.87 \AA^{-1}$. The 1 s core states were obtained by solving the Dirac equation, while spin-orbit coupling for the valence electrons was treated within the muffin-tin radius of 1.34 a.u. by the second 
variational method [19]. Finally, external transverse electric field was included as a periodic zigzag electric potential added to the exchange-correlation functional [20].

Our main results are shown in Fig. 2 which displays a variety of the band structure topologies of graphene, tunable by electric field. The intrinsic case (zero external field) shows a splitting of the Dirac cones at the $K\left(K^{\prime}\right)$ points into two "rounded" cones, with the gap of $2 \lambda_{I}=24 \mu \mathrm{eV}(0.28 \mathrm{~K})$. The bands are two-fold degenerate due to the presence of time reversal and space inversion symmetries [21]. Transverse electric field breaks the latter symmetry, resulting in a spin-splitting $2 \lambda_{\mathrm{BR}}$ of the energy levels: for each momentum at a given band there are two states with energy differing by $2 \lambda_{\mathrm{BR}}$. This extrinsic splitting is akin to the Bychkov-Rashba (BR) spinorbit coupling in semiconductor heterostructures [22].

The band structure of graphene in the presence of transverse electric field depends rather strongly on the interplay of the intrinsic and extrinsic spin-orbit coupling effects. As the magnitude of the electric field increases, we encounter the topologies on display in Fig. 22 If the $\mathrm{BR}$ splitting is lower than the intrinsic one, the spectral gap gets smaller. The electron branch of the spectrum is still degenerate at $K\left(K^{\prime}\right)$; in contrast, the hole branch is split by $2 \lambda_{\mathrm{BR}}$. This topology can give a quantum spin Hall insulator [8]. Curiously, as the electric field is such that $\lambda_{\mathrm{BR}}=\lambda_{\mathrm{I}}$, one of the hole branches rises, forming a genuine touching cones structure of massless fermions with one of the electron branches. The two remaining branches are parabolic (massive). For fields such that $\lambda_{\mathrm{BR}}>\lambda_{\mathrm{I}}$, all the branches are again parabolic, with a degeneracy of one electron and one hole band. The calculated spectrum at $K\left(K^{\prime}\right)$ follows the recipe $\mu \lambda_{\mathrm{BR}}+\nu\left|\lambda_{\mathrm{BR}}-\mu \lambda_{\mathrm{I}}\right|$, with $\mu$ and $\nu$ being \pm 1 .

The physics behind the calculated spectral topologies can be described qualitatively by previously proposed effective Hamiltonians. Without spin-orbit coupling the electronic band structure of graphene in the vicinity of $K\left(K^{\prime}\right)$ is described by the Hamiltonian $\mathcal{H}_{0}=$ $\hbar v_{\mathrm{F}}\left(\kappa \sigma_{x} k_{x}+\sigma_{y} k_{y}\right)$. Here $v_{\mathrm{F}}$ is the Fermi velocity, $k_{x}$ and $k_{y}$ are the cartesian components of the electron wave vector measured from $K\left(K^{\prime}\right)$, the parameter $\kappa=1(-1)$ for the cones at $K\left(K^{\prime}\right)$, and $\sigma_{x}$ and $\sigma_{y}$ are the Pauli matrices acting on the so called pseudospin space formed by the two triangular sublattices of graphene. The Hamiltonian $\mathcal{H}_{0}$ describes gapless states with conical dispersion $\varepsilon_{0}=\nu \hbar v_{\mathrm{F}}|\boldsymbol{k}|$ near the Dirac points. The eigenstates are $\psi_{\nu}=1 / \sqrt{2}\left|\nu e^{-i \kappa \varphi}, \quad 1\right\rangle$ for the electron band, $\nu=1$, and hole band, $\nu=-1$.

The intrinsic spin-orbit coupling is described by the effective Hamiltonian $\mathcal{H}_{\mathrm{SO}}=\lambda_{\mathrm{I}} \kappa \sigma_{z} s_{z}\left[8\right.$, , 15]. Here $s_{z}$ is the spin Pauli matrix. The spin-orbit coupling lifts the orbital degeneracy at $K\left(K^{\prime}\right)$. Indeed, the eigenvalues of the combined Hamiltonian, $\mathcal{H}_{0}+\mathcal{H}_{\mathrm{SO}}$, are $\varepsilon_{\nu}=\nu \sqrt{\varepsilon_{0}^{2}+\lambda_{\mathrm{I}}^{2}}$. The bands are split by $2\left|\lambda_{I}\right|$, but the two-fold degeneracy of the bands, required by space inversion and time reversal symmetry, remains. The eigenvectors are $\psi_{\mu \nu}=\chi_{\mu}\left|e^{-i \kappa \varphi}\left(\kappa \varepsilon_{\nu}+\mu \lambda_{\mathrm{I}}\right) / \varepsilon_{0}, \quad 1\right\rangle / C_{\mu \nu}$. Here $\chi_{\mu}$ is the spin spinor with $\mu= \pm 1$; the normalization constant is $C_{\mu \nu}=\left[1+\left(\kappa \varepsilon_{\nu}+\mu \lambda_{\mathrm{I}}\right)^{2} / \varepsilon_{0}^{2}\right]^{1 / 2}$.

The extrinsic spin-orbit coupling of the BychkovRashba type in graphene can be described by the Hamiltonian $\mathcal{H}_{\mathrm{BR}}=\lambda_{\mathrm{BR}}\left(\kappa \sigma_{x} s_{y}-\sigma_{y} s_{x}\right)$, where $\lambda_{\mathrm{BR}}$ is the Bychkov-Rashba parameter [8]. Unlike in semiconductor heterostructures, the coupling in graphene does not depend on the magnitude of the electron momentum, as the electrons at $K\left(K^{\prime}\right)$ have a constant velocity. The electronic bands near $K\left(K^{\prime}\right)$ are now modified to

$$
\varepsilon_{\mu \nu}=\mu \lambda_{\mathrm{BR}}+\nu \sqrt{\left(\hbar v_{\mathrm{F}} k\right)^{2}+\left(\lambda_{\mathrm{BR}}-\mu \lambda_{\mathrm{I}}\right)^{2}} .
$$

The corresponding eigenvectors are

$$
\begin{aligned}
& \psi_{\mu \nu}=\left(\chi_{-}\left|\kappa e^{-i \kappa \varphi}\left[\left(\varepsilon_{\mu \nu}-\lambda_{\mathrm{I}}\right) / \varepsilon_{0}\right]^{\kappa}, \quad 1\right\rangle+\right. \\
& \left.\mu \chi_{+}\left|-i \kappa e^{-i(1+\kappa) \varphi}, \quad i e^{-i \varphi}\left[\left(\lambda_{\mathrm{I}}-\varepsilon_{\mu \nu}\right) / \varepsilon_{0}\right]^{\kappa}\right\rangle\right) / C_{\mu \nu},
\end{aligned}
$$

with the normalization constant $C_{\mu \nu}=\sqrt{2}\left(1+\left[\left(\lambda_{\mathrm{I}}-\right.\right.\right.$ $\left.\left.\left.\varepsilon_{\mu \nu}\right) / \varepsilon_{0}\right]^{2 \kappa}\right)^{/ 2}$. The expectation value of the spin,

$$
s_{\mu \nu}=\frac{\varepsilon_{0}}{\sqrt{\varepsilon_{0}^{2}+\left(\lambda_{\mathrm{I}}-\mu \lambda_{\mathrm{BR}}\right)^{2}}}\left(\begin{array}{c}
\sin \varphi \\
-\cos \varphi \\
0
\end{array}\right),
$$

is $k$-dependent and lies in the graphene plane. The inclusion of the extrinsic coupling lifts the two-fold degeneracy of the bands. Only the time-reversal Kramers degeneracy remains, coupling states at $K$ and $K^{\prime}$.

Our first-principles results show that the above effective Hamiltonian model gives a remarkably faithful description of graphene's band structure at $K\left(K^{\prime}\right)$. The comparison is shown in Fig. 2. The dispersions given by Eq. (1) differ from the numerical results by less than $5 \%$ up to $\pm 200 \mathrm{meV}$ away from the Fermi level. With the parameters supplied by the first-principles calculations the analytical model becomes highly accurate.

The extrinsic splitting $2 \lambda_{\mathrm{BR}}$ is extracted as $\left(\varepsilon_{+-}-\right.$ $\left.\varepsilon_{--}\right) / 2$ for $\lambda_{\mathrm{BR}}<\lambda_{\mathrm{I}}$, and $\left(\varepsilon_{++}-\varepsilon_{--}\right) / 2$ for $\lambda_{\mathrm{BR}}>\lambda_{\mathrm{I}}$, at $K$. Figure 3(a) illustrates the zigzag potential modeling the transverse field. The calculated $2 \lambda_{\mathrm{BR}}$ versus the electric field $E$ is shown in Fig. 3(b). The dependence is linear with the slope of $10 \mu \mathrm{eV} \mathrm{nm} / \mathrm{V}$. Since the field of $1 \mathrm{~V} / \mathrm{nm}$ is produced by an electron charge $1 \mathrm{~nm}$ away from the graphene sheet, such fields are typical for graphene on a substrate. We expect that in realistic situations the intrinsic and extrinsic spin-orbit couplings compete, making the topologies described in Fig. 2 likely occurring in real samples. Since the extrinsic coupling depends linearly on the electric field, the topology is tunable by gates. We also give the calculated magnitude of the graphene's dipole moment (the shift of the electron charge density): 0.0134 CA in unit cell. One may then relate the Bychkov-Rashba effect directly to the induced 
dipolar moment; this should be particularly useful for estimating the extrinsic splitting due to ad-atoms absorption on graphene.

Previous numerical estimates for the intrinsic splitting $2 \lambda_{\mathrm{I}}$ in graphene are rather controversial. The splitting was estimated to be in the range of 1 to $200 \mu \mathrm{eV}$ [8, 23, 24, 25, 26]. Kane and Mele [8] estimated the splitting of $200 \mu \mathrm{eV}$. This optimistic estimate was drastically reduced by Min et al. 23] to the value of $1 \mu \mathrm{eV}$, supported by subsequent works [24, 25]. None of these studies were fully first-principles. A density functional calculation of Boettger and Trickey [26], using a Gaussiantype orbital fitting function methodology, gave $50 \mu \mathrm{eV}$. Our result is about one half of that; the difference is likely due to the different approximation schemes for spin-orbit coupling used in Ref. 26 and by us 29]. Previous estimates for the extrinsic splitting, $2 \lambda_{\mathrm{BR}}$, are $0.516 \mu \mathrm{eV}$ [8] and $133 \mu \mathrm{eV}$ 23] per $\mathrm{V} / \mathrm{nm}$. No fully first-principles calculation of $2 \lambda_{\mathrm{BR}}$, or the extrinsic effects in graphene in general, was reported thus far.

What is the origin of the rather large, as compared to previous non-fully-first-principles results, intrinsic spinorbit splitting in graphene? We calculate the spin-orbit coupling splitting of the $2 p$ levels in carbon atoms to be $8.74 \mathrm{meV}$, using the Wien2k code. This splitting should be reflected in the splitting of the bands at the $\Gamma$ point. Our calculation finds the splitting at the $\Gamma$ point of $8.978 \mathrm{meV}$ about $3 \mathrm{eV}$ below the Fermi level, in close agreement with the atomic value. The bands at the $K\left(K^{\prime}\right)$ points are formed mainly by $p_{z}$ orbitals whose magnetic quantum number is zero. The intrinsic splitting can be due to the coupling of the $p_{z}$ orbitals (forming the $\pi$-bands) to either $\sigma$ bands or bands formed by higher orbitals $(d, f, \ldots)$. As argued already by Slonczewski 16] using group theory, it is the $d$ and higher orbitals that dominate the spin-orbit splitting at $K\left(K^{\prime}\right)$. A qualitative argument for that was provided by McClure and Yafet [15]: Orbitals $d_{x z}$ and $d_{y z}$ can form Bloch states of the $\pi$ band symmetry at $K\left(K^{\prime}\right)$. Due to a finite overlap between the neighboring $p_{z}$ and $d_{x z}, d_{y z}$ orbitals, the intrinsic splitting is linearly proportional to the spin-orbit splitting of the $d$ states (orbitals higher than $d$ have a smaller overlap and contribute less). In contrast, due to the absence of the direct overlap between the $p_{z}$ and $\sigma$ band orbitals, the usually considered [8, 23, 24, 25] spinorbit splitting induced by the $\sigma-\pi$ mixing depends only quadratically on the atomic spin-orbit splitting, giving a negligible contribution.

In Fig. 3(c) we show the orbital-resolved densities of states. The $p_{x}, p_{y}$ atomic character vanishes above $-3 \mathrm{eV}$ (the Fermi level $\varepsilon_{\mathrm{F}}$ is at zero). The density of states close to $\varepsilon_{\mathrm{F}}$ comes predominantly from $p_{z}$ orbitals $(\pi$ bands). Nevertheless, there is a finite contribution from $d$ orbitals $\left(d_{x z}\right.$ and $\left.d_{y z}\right)$ which follows in shape that of $p_{z}$. The contributions from $d_{x^{2}-y^{2}}, d_{x y}$, and $d_{z^{2}}$ vanish for energies above the $-3 \mathrm{eV}$. To further confirm the symmetry arguments of Slonczewski, we have selectively removed orbitals from the calculation of the spin-orbit coupling contribution. Removing $d$ and higher orbitals reduces the intrinsic splitting to $0.98 \mu \mathrm{eV}$, reproducing earlier non-first-principles calculations [23, 24, 25]. We conclude that the intrinsic splitting is dominated by $d$ and higher orbitals, giving more that $96 \%$ of the splitting [30]. In contrast, we find that the extrinsic BR coupling is largely unaffected by the presence of $d$ and higher orbitals, demonstrating that this coupling is due to the $\sigma-\pi$ mixing.

Finally, we calculate the spin-orbit splitting for what we call a 'mini-ripple' configuration (Fig. (4), in which every other atom is displaced by an amount $\Delta$ transverse to the sheet. This calculation should give an indication of what to expect for larger-scale ripples that occur in graphene on a substrate [27] or free standing [28]; such large scales are out of the scope for our methods. The mini-ripple exhibits a gap that grows quadratically with increasing $\Delta$, as seen from Fig. 4 Removing $d$ and higher orbitals from the calculation of the spin-orbit splitting, the initial gap reduces to about $1 \mu \mathrm{eV}$ but the overall growth remains largely unchanged: the rippling-induced gap is almost solely due to $\sigma-\pi$ mixing. Furthermore, the displacements of less than $1 \%$ have no significant effects on the intrinsic spin-orbit splitting. For an effective description of the gap opening in the mini-ripple we consider a more general extrinsic case. The contribution of a transverse electric field is twofold. First, there is a pseudospin splitting, as the two sublattices are no longer equivalent (the two triangular sublattices have a different potential). Second, the Bychkov-Rashba effect appears. We find that the following formula describes the resulting spectrum: $\epsilon_{\mu \nu}^{K}=\nu \lambda_{\mathrm{I}}+\mu\left[\delta_{1 \nu} \lambda_{\Delta}+\delta_{-1 \nu} \sqrt{\lambda_{\Delta}^{2}+\left(2 \lambda_{\mathrm{BR}}\right)^{2}}\right]$, where $\lambda_{\Delta}$ is the electric field induced gap due to the rippling $\left(\lambda_{\Delta} \propto E\right)$.

In summary, we have shown that for realistic electric fields the graphene band structure exhibits remarkable tunable topologies. Our first-principles calculation gives strong support for the effective spin-orbit coupling Hamiltonian models, making them highly accurate analytical tools to investigate the physics of graphene.

We thank A. Matos-Abiague, P. Blaha, S. B. Trickey, and the Wien2k community for useful hints and discussions. This work was supported by the DFG SFB 689 and SPP 1285. CAD appreciates support from the Austrian Science Fund, Project I107.

[1] K. S. Novoselov, et al., Science 306, 666 (2004).

[2] K. S. Novoselov, et al., Nature 438, 197 (2005).

[3] Y. Zhang, Y.-W. Tan, H. L. Stormer, P. Kim, Nature 438, 201 (2005).

[4] A. Bostwick, T. Ohta, T. Seyller, K. Horn, E. Rotenberg, Nature Phys. 3, 36 (2007). 
[5] A. K. Geim, K. S. Novoselov, Nature Mat. 6, 183 (2007).

[6] S. Adam, E. H. Hwang, V. M. Galitski, S. Das Sarma, PNAS 104, 18392 (2007).

[7] N. Tombros, C. Jozsa, M. Popinciuc, H. T. Jonkman, B. J. van Wees, Nature 448, 571 (2007).

[8] C. L. Kane, E. J. Mele, Phys. Rev. Lett. 95, 226801 (2005).

[9] B. Trauzettel, D. V. Bulaev, D. Loss, G. Burkard, Nature Phys. 3, 192 (2007).

[10] P. R. Wallace, Phys. Rev. 71, 622 (1947).

[11] C. A. Coulson, R. Taylor, Proc. Phys. Soc. A 65, 815 (1952).

[12] W. M. Lomer, Proc. Roy. Soc. (London) A227, 330 (1955).

[13] J. W. McClure, Phys. Rev. 108, 612 (1957).

[14] J. C. Slonczewski, P. R. Weiss, Phys. Rev. 109, 272 (1958).

[15] J. W. McClure, Y. Yafet, Proceedings of the Fifth Conference on Carbon (Pergamon, New York, 1962), vol. 1, pp. 22-28.

[16] J. C. Slonczewski, Band theory of graphite, Ph.D. thesis, Rutgers University of New Jersey (1955).

[17] P. Blaha, K. Schwarz, G. Madsen, D. Kvasnicka, J. Luitz, WIEN2k - An Augmented Plane Wave Plus Local Orbitals Program for Calculating Crystal Properties, TU Wien, Austria (2008).

[18] J. P. Perdew, K. Burke, M. Ernzerhof, Phys. Rev. Lett. 77, 3865 (1996).

[19] D. J. Singh, L. Nordstrom, Planewaves, Pseudopotentials, and $L A P W$ method (Springer, 2005).
[20] J. Stahl, U. Pietsch, P. Blaha, K. Schwarz, Phys. Rev. B 63, 165205 (2001).

[21] J. Fabian, A. Matos-Abiague, C. Ertler, P. Stano, I. Žutić, Acta Phys. Slov. 57, 565 (2007).

[22] Y. A. Bychkov, E. I. Rashba, JETP Lett. 39, 78 (1984).

[23] H. Min, et al., Phys. Rev. B 74, 165310 (2006).

[24] Y. Yao, F. Ye, X.-L. Qi, S.-C. Zhang, Z. Fang, Phys. Rev. $B$ 75, 041401 (2007).

[25] D. Huertas-Hernando, F. Guinea, A. Brataas, Phys. Rev. $B$ 74, 155426 (2006).

[26] J. C. Boettger, S. B. Trickey, Phys. Rev. B 75, 121402 (2007).

[27] M. Ishigami, J. H. Chen, W. G. Cullen, M. S. Fuhrer, E. D. Williams, Nano Lett. 7, 1643 (2007).

[28] A. Fasolino, J. H. Los, M. I. Katsnelson, Nature Mat. 6, 858 (2007).

[29] The precise value of the intrinsic gap depends on the muffin-tin radius, since Wien2k calculates spin-orbit coupling only inside the muffin-tin sphere. (The present calculations use the maximum allowed sphere radius.) Although spin-orbit coupling is concentrated around the atom cores, making the inner-sphere approximation rather accurate, it is likely that the actual value of the gap is somewhat larger.

[30] The contribution to $\lambda_{\mathrm{I}}$ changes nonmonotonic as the maximum orbital state $\ell_{\max }$ in the second variational scheme is increased. For $\ell_{\max }=1,2$, and 3 and more, we get $2 \lambda_{\mathrm{I}} \approx 1,26$, and $24 \mu \mathrm{eV}$. 


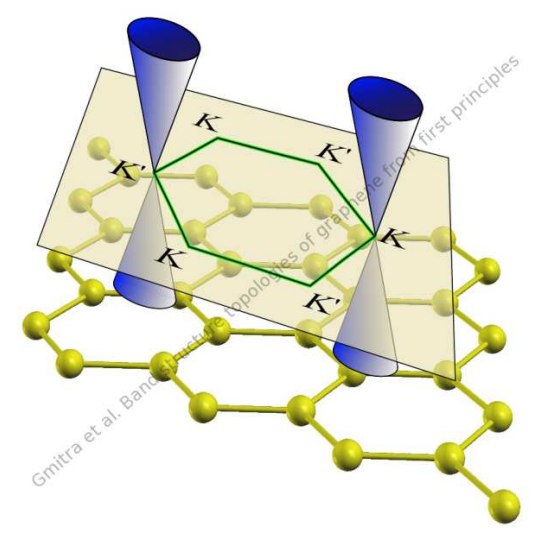

FIG. 1: Graphene's essentials. Bottom-up: Carbon atoms form a honeycomb lattice with two atoms in the unit cell. The first Brillouin zone of the reciprocal lattice contains two nonequivalent Dirac points, $K$ and $K^{\prime}$. The relevant states at the Fermi level form two touching cones with the tips at $K\left(K^{\prime}\right)$.
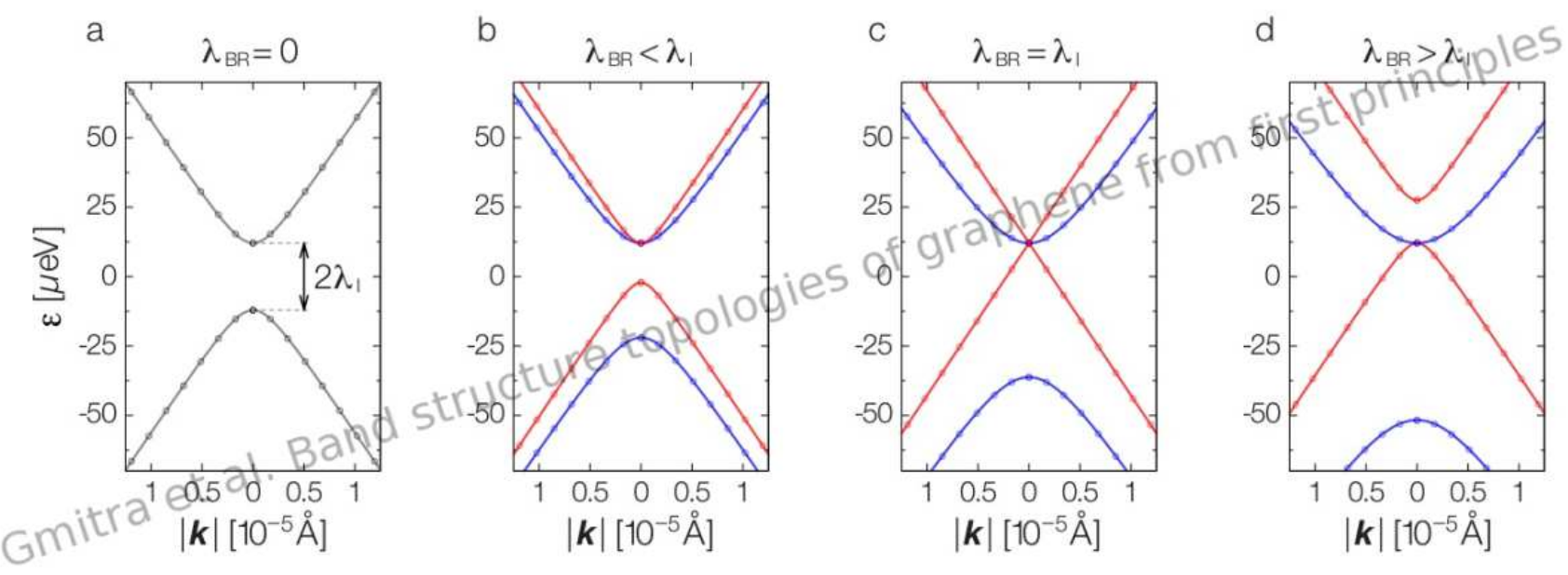

FIG. 2: Band structure topologies of graphene. Transverse electric field drastically changes the topology of the bands near $K\left(K^{\prime}\right)$. (a) Zero electric field. (b) Electric field of magnitude $E=1.0 \mathrm{~V} / \mathrm{nm}$. (c) $E=2.44 \mathrm{~V} / \mathrm{nm}$. (d) $E=4.0 \mathrm{~V} / \mathrm{nm}$. The first-principles results are represented by circles (the Fermi level is at zero). The curves are fits to the analytical model (see the main text). The spin branch $\mu=1$ is shown in red, $\mu=-1$ in blue. The calculated Fermi velocity is $v_{\mathrm{F}}=0.833 \times 10^{6} \mathrm{~m} / \mathrm{s}$. 
a

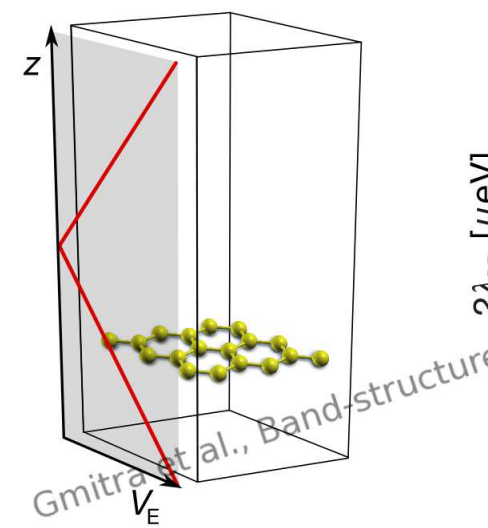

b

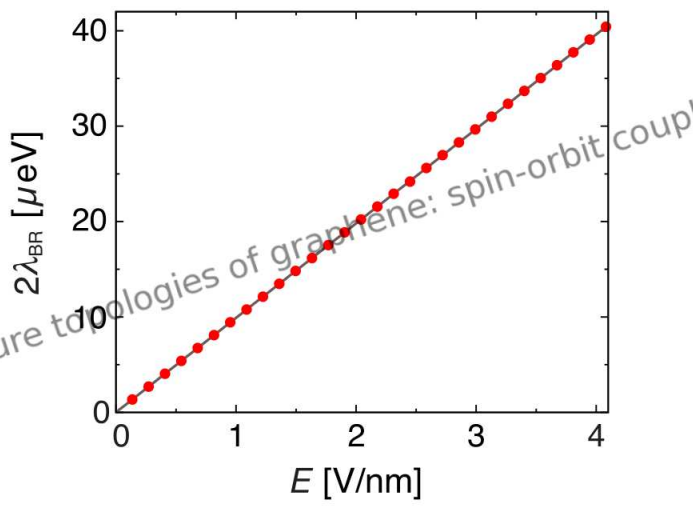

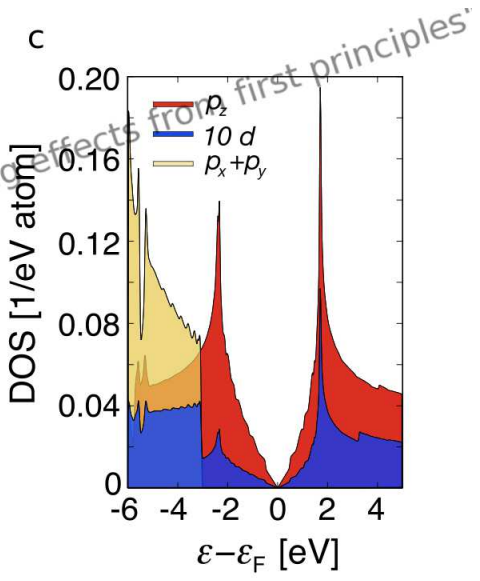

FIG. 3: Bychkov-Rashba-type splitting in graphene and orbital-resolved density of states. (a) Transverse electric field is modeled with a zigzag potential. (b) Bychkov-Rashba spin-orbit induced splitting at $K\left(K^{\prime}\right)$ as a function of the electric field. The slope is $9.9 \mu \mathrm{eV}$ per $\mathrm{V} / \mathrm{nm}$. (c) Projected density of states to particular atomic orbitals. The Fermi level is at zero. The $d$-character is enhanced by a factor of ten.

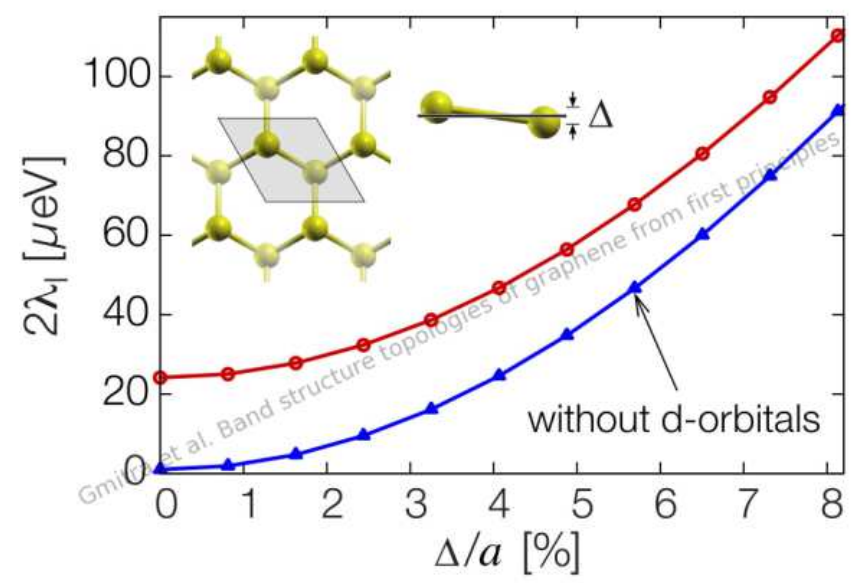

FIG. 4: Calculated spectral gap at $K\left(K^{\prime}\right)$ in the mini-ripple configuration (inset), as a function of the relative corrugation strain with respect to the lattice constant $a$. Neglecting $d$ and higher orbitals results in an almost constant shift, proving that the main effects come from $\sigma-\pi$ hybridization. 